\title{
5 Von „Trade, not Aid“ zu „Weniger nehmen“
}

\subsection{Die Professionalisierung der Fair-Trade-Bewegung}

Die Geschichte der Wandlung der Idee eines gerechten Nord-Süd-Handels in der Dritte-Welt-Bewegung ist in erster Linie eine Geschichte der Entakademisierung und der Professionalisierung. Mit dem Übergang von der ehrenamtlichen und aktivistischen Bewegung von Akademikern zu professionellen Strukturen fand gleichzeitig ein Abschied vom Grundsatz der Wissenschaftlichkeit statt. Dieser Grundsatz hatte für die Aktivistinnen und Aktivisten der Anfangszeit unausgesprochen Gültigkeit, da es sich bei ihnen zu einem grossen Teil um Professoren und Universitätsdozenten gehandelt hatte. In den ersten Jahren der Professionalisierung setzte sich Rudolf Strahm noch aktiv und mühsam für die akademischen Grundlagen der Kampagnen ein. „Zu dieser Zeit fühlten wir uns verpflichtet, jeder politischen Aktion auch eine wissenschaftliche Fundierung zu geben“, schrieb er und weist mit seiner Formulierung „zu dieser Zeit“ darauf hin, dass die wissenschaftliche Fundierung in der Folge vernachlässigt wurde. ${ }^{1}$ Doch bereits Strahm entfernte sich in seiner Zeit als Sekretär der EvB von seinen wissenschaftlichen Wurzeln. Nach seinem Weggang gab es innerhalb der Bewegung niemanden, der aktiv den akademischen Rückhalt gefordert hätte. Wie aufgezeigt, haben Naturwissenschaftlerinnen und -wissenschaftler den Abschied von der akademischen Makroökonomie und den Widerspruch zwischen wirtschaftlicher Entwicklung und Umweltschutz mit ihrem Engagement übertüncht. Die Abkopplung des kleinen Fachbereiches development studies von der ursprünglichen Verankerung in der Entwicklungsökonomie kreierte zudem dort eine kleine Gruppe von Experten, die einen Kulturbegriff portierten, der die Widersprüchlichkeit von Entwicklungshilfe und Neoprotektionismus als Wertepluralität interpretierte und Mitte der 1970er-Jahre auch direkt die UNCTAD kritisierte. ${ }^{2}$ Mit der Professionalisierung der spontanen Bewegung folgte auch ein aktives und konstantes Suchen nach Betroffenheit, nach Themen und Kampagnen, um die professionelle Organisation am Leben zu erhalten. Damit verlor sich die spontane Bewegung, die auf Forderungen der in der UNCTAD zusammengeschlossenen Entwicklungsländer lediglich reagiert hatte. Die aktive Rolle der Entwicklungsländer und das Verständnis der frühen Dritte-Welt-Bewegung, als Advokat dieser Länder im Norden aufzutreten, verschwand. Die westliche Dritte-Welt-Bewegung wurde zum professionellen Sektor, der auf der Spendergunst im Westen basierte.

1 A.-M. Holenstein-Hasler, R. Renschler, R. H. Strahm: Entwicklung heisst Befreiung, 127.

2 A. Bänziger: Entwicklungsland Welt - Entwicklungsland Schweiz, 34.

๖ OpenAccess. (๑) 2020 Andrea Franc, publiziert von De Gruyter. (cc) BY-Nc-ND Dieses Werk ist lizenziert unter der Creative Commons Attribution-NonCommercial-NoDerivatives 4.0. 
Die Entwicklungsländer wurden für NGOs zur Folie, die sie der Spenderin oder dem Spender präsentierten. ${ }^{3}$

Die Professionalisierung der Bewegung führte auch zur Bildung von Allianzen. Mittels Diskursbrücken einerseits, aber auch mit einer mittelfristig grundlegenden Änderung ihres Konzepts ging die Bewegung Allianzen ein mit der Umweltbewegung, den Gewerkschaften, den inländischen Kleinbauern, Konsumentenverbänden und Frauengruppen. Eine Brücke zur breiten Gruppe der kirchlich engagierten Bürgerlichen war gar nicht mehr nötig, da Letztere bereits von Beginn weg zur Basis der EvB gehört hatten. Eine radikal-sozialistische, linke Befreiungsbewegung war nie Teil der Fair-Trade-Bewegung gewesen, daher musste sich Erstere auch nicht von einer solchen entfernen. Das merkwürdige Aussenstehen des sozialistischen Nationalrats Jean Ziegler zeigt dies auf.

Spätestens Ende der 1970er-Jahre repräsentierte die Fair-Trade-Bewegung als Teil einer grösseren sozialen Bewegung, zu der auch die Bio-Bewegung gehörte, eine breite Bevölkerungsschicht. Dies zeigt sich etwa in der Lancierung des Vereins M-Frühling durch Migros-Genossenschafterinnen und -genossenschafter ab 1979. ${ }^{4}$ Nachdem die EvB ihre Fair-Trade-Verkäufe 1977 an eine private Firma outgesourct hatte, wurde sie zu einem paraakademischen Think-Tank. Sie produzierte vor allem Informationen, bildete aus und trat im gesellschaftlichen und politischen Diskurs als intellektuelle Vertreterin der Bio- und Fair-Trade-Bewegung auf. ${ }^{5}$ Die aus den Zeiten der Ehrenamtlichkeit der 1960er-Jahre stammende moralische Autorität wurde zum Startkapital, das in die professionelle Zukunft führte.

Gemäss Körber ist genau diese moralische Autorität das Kapital der NGOs. Die moralische Autorität ist nicht nur die Grundlage für Spenderinteressen, sondern die Basis der eigentlichen Aktivität der NGO. ${ }^{6}$ Dank ihrer moralischen Autorität kann eine NGO die öffentliche Meinung beeinflussen und wird zu einem bedeutenden, noch viel zuwenig untersuchten Akteur im demokratischen Prozess. ${ }^{7}$ So können NGOs die Reputation von Konzernen schädigen oder Parlamentsentscheide beeinflussen. Diese moralische Autorität wird zunächst in einer aktivistischen, ehrenamtlichen Phase aufgebaut. Im Falle der EvB folgte darauf eine halb ehrenamtliche Phase, in der die ersten Vertreterinnen und Vertreter zwar ein kleines Einkommen erhielten, sich aber weit über ihre Bezahlung hinaus enga-

3 M. Hilton: Politics of Expertise, 256, siehe auch A. Müller, F. Rauh: Wahrnehmung und mediale oder M. Jennings: ,Almost an Oxfam in Itself‘.

4 B. Mahler: ,umdenken - umschwenken'.

5 K. J. Kuhn: Entwicklungspolitische Solidarität, 257, P. Moser: Stand der Bauern, 311-312.

6 A. Körber: The Political Economy. Siehe auch M. Hilton: Politics of Expertise, 56.

7 Ebd., 254. 
gierten und von ehrenamtlichen Mitarbeitern unterstützt wurden. Darüber hinaus erweckten die ersten, bezahlten Angestellten auch weiterhin einen ehrenamtlichen Eindruck. Die frühen NGO-Angestellten mussten die professionellen Strukturen und ein professionelles Verhalten noch erarbeiten. Die Tatsache, dass die professionelle NGO mit ihren bezahlten Angestellten aus einer spontanen sozialen Bewegung entstand und weiterhin in dieser Bewegung verankert war, verlieh der NGO zudem moralische Autorität. Die NGOs stellen ihre moralische Autorität gerne dadurch her, dass sie ihre eigene Geschichtsschreibung pflegen. Gerne verweisen hochprofessionelle NGOs auf ihre Ursprünge in einer sozialen, ehrenamtlichen Bewegung, in den Graswurzeln. ${ }^{8}$

Interessanterweise lässt sich gemäss Hilton bei vielen NGOs nach einer ersten Aufbauphase ein personeller Wechsel feststellen. Gemäss dem typischen Muster einer NGO traten die charismatischen, eigenwilligen Aufbauarbeiter der 1960erund 70er-Jahre nach einiger Zeit verärgert zurück oder wurden verdrängt, um einer professionellen Struktur Platz zu machen. ${ }^{9}$ Im Falle der EvB machten die ehrenamtlich arbeitenden Pfarrer und Theologieprofessoren bereits zu Beginn der 1970er-Jahre freiwillig den Frauen und den Jungen Platz, die zwar angestellt und bezahlt waren, sich aber noch als Aktivistinnen und Aktivisten verstanden und es als selbstverständlich erachteten, für den Bruchteil eines marktüblichen Lohns bis zur Erschöpfung zu arbeiten. Als Rudolf Strahm 1978 die EvB verliess, musste er gleich durch zwei Nachfolger ersetzt werden, da er bis zu 70 Stunden pro Woche gearbeitet hatte. ${ }^{10} 1982$ kündigte Anne-Marie Holenstein, 1985 Regula Renschler. Orchestriert wurden beide Abgänge durch vorangegangene interne „Spannungen“. ${ }^{11}$ Die Kündigung von Anne-Marie Holenstein fiel zusammen mit rigorosen Sparmassnahmen aufgrund des finanziellen Verlustes der von ihr geleiteten, mehrjährigen Kampagne „Hunger ist ein Skandal“. ${ }^{12}$ Hilton nennt dieses bei mehreren NGOs festzustellende Muster founder's syndrome, das Gründer-Syndrom. ${ }^{13}$ Während die schillernden Gründer intern oft einer professionellen Angestelltengruppe Platz machen mussten, waren diese Persönlichkeiten weiterhin wichtig für den Fokus auf die „Gründungsmythen“. Die moralische Autorität der NGO, die durch die „Gründungsmythen“ symbolisiert wird, bildet die Entscheidungsgrundlage für die Spender. Dies umso mehr, als Hilton im Rahmen des

8 Ebd., 56.

9 Ebd., 68.

10 A.-M. Holenstein-Hasler, R. Renschler, R. H. Strahm: Entwicklung heisst Befreiung, 204.

11 Ebd., 206.

12 SozArch, EvB, Ar 430.90.1. Jahresberichte 1978-2003. EvB: Jahresbericht 1983, S. 14. K. J. Kuhn: Entwicklungspolitische Solidarität, 268-269.

13 M. Hilton: Politics of Expertise, 68. 
DANGO-Projekts für Grossbritannien festgestellt hat, dass NGOs zunehmend Kirchen und politische Parteien ersetzen. ${ }^{14}$ Spontane Bewegungen und die daraus entstehenden professionellen NGOs boten auch in der Schweiz ein „Auffangbecken“ in der Zeit der massenhaften Kirchenaustritte der 1960er-Jahre und der Politikverdrossenheit der 1980er-Jahre. ${ }^{15}$ In diesem Sinne spricht Hilton von pressure groups, also zivilgesellschaftlichen Bewegungen, die sich ausserhalb bzw. am Rande des politischen Prozesses für gewisse Anliegen einsetzen. Mit pressure group benutzt Hilton lediglich ein Synonym des politökonomischen Fachbegriffs special interest groups. Wie im DANGO-Projekt festgestellt wurde, ist in Grossbritannien die Zahl der Mitglieder von Kirchen und politischen Parteien genau wie die Wahlbeteiligung rückläufig, während NGOs Mitglieder gewinnen. Gemäss Studien hat das öffentliche Vertrauen in die Regierungen abgenommen, jenes in NGOs jedoch zugenommen. ${ }^{16}$ Dies ist aber eine deskriptive Beobachtung. Die Frage ist nun, ob die NGOs profitiert haben von einem abnehmenden Vertrauen in die Regierung oder ob sie dieses Misstrauen und ihre eigene moralische Autorität aktiv aufgebaut haben. Was den Fair-Trade-Konsum angeht, stellt Anderson jedenfalls fest: „It is clear that ethical consumers did not emerge but were shaped through the campaigns and experience of alternative trade pioneered by NGOs. "17 Malinowski und Sedlmaier vertreten die These, dass die Kulturrevolution von 1968 erheblich dazu beigetragen hat, einen neuen Konsumententypus zu schaffen. ${ }^{18}$ Trentmann weist wiederum darauf hin, dass Kampagnen für ethischen Konsum Jahrzehnte vor 1968 an der Tagesordnung waren und aufgrund ihrer teilweise umgekehrten politischen Stossrichtung von der Forschung übersehen worden seien. ${ }^{19}$

Während NGOs oft weiterhin für die Spender den Anschein einer uneigennützigen sozialen Bewegung erwecken, haben sich die Vorzeichen aus politökonomischer Sicht fundamental geändert. Das Personal der professionellen NGOs besteht nun aus bezahlten Angestellten, deren Einkommen von der Gunst der Spender abhängt. Auch ist davon auszugehen, dass die Vertreter der NGOs analog zu Unternehmern und Bürokraten wenn nicht das Wachstum, so doch zumindest

14 M. Hilton: A Historical Guide, 326.

15 U. Hofmann: Innenansichten eines Niedergangs, 262.

16 M. Hilton: A Historical Guide, 326.

17 M. Anderson: Fair Trade: NGOs, 236.

18 Malinowski, Stephan; Sedlmaier, Alexander: ,1968‘ als Katalysator der Konsumgesellschaft. Performative Regelverstösse, kommerzielle Adaptionen und ihre gegenseitige Durchdringung, in: Geschichte und Gesellschaft 32/2 (2006) 238-269, 239.

19 F. Trentmann: Before Fair Trade. 
den Erhalt ihrer Organisation und damit ihrer eigenen Stelle zum Ziel haben. ${ }^{20}$ Ausgehend von dieser Annahme scheint es nur eine logische Folge, dass die EvB, nachdem sie von einer sozialen Bewegung in eine professionelle NGO überführt wurde, ihr Konzept dahingehend änderte, dass es eine möglichst breite Gruppe von Spenderinnen und Spendern ansprach. Eine Bedingung für ihr Organisationswachstum bestand darin, dass sie ihre Ideologie von umstrittenen Punkten befreite und sich auf die kleinste gemeinsame Schnittmenge konzentrierte, die ihre zahlreichen potentiellen Spendergruppen hatten. Der Kleinbauer im In- und Ausland füllte diese gemeinsame Schnittmenge aus.

Der Aktivismus der 1960er-Jahre wurde für die professionellen NGOs zum Produkt. Eine selbstkritische Hinterfragung der eigenen Rolle, des eigenen Wachstums und der Grösse der Organisation wurde im Rahmen dieses Strukturwandels für eine professionell funktionierende Entwicklungs-NGO zur Unmöglichkeit. Nicht nur die Selbstreflexion als professionelle NGO, auch die grundsätzliche Systemkritik, die zur Kritik an der Spenderin oder am Spender geführt hätte, wurde zur Unmöglichkeit. Denn die Kritik an der Spenderin und dem Spender hätte zum Ausbleiben der Spenden und damit zur Abschaffung der NGO selbst geführt. Die System- und Selbstkritik, wie sie im Manifest von 1968 geäussert worden war, brachte für die professionelle NGO nur Nachteile. Das neue Fair-Trade-Konzept des Kleinbauern hingegen bot der NGO zahlreiche Vorteile. Es ging von einem utopisch-statischen Bild des Kleinbauern aus, das auf einer jahrhundertealten, wenn nicht biblischen Moralvorstellung des Menschen als einem sesshaften Bauern auf seinem Ackerland beruhte. Dieses Bild des Kleinbauern war universell, es konnte beliebig für die Schweiz wie für die Dritte Welt eingesetzt werden. Zudem beinhaltete die Konzentration auf den Kleinbauern die Repetition des ewiggleichen, statischen Bildes der Feldarbeit. Damit konnte die NGO gerade in Zeiten rasanter wirtschaftlicher Veränderungen, Innovationen und Wachstumsphasen ein Erkennungsmerkmal vorweisen, das nicht mit den faktischen wirtschaftlichen Veränderungen in der Dritten Welt, etwa dem industriellen Aufschwung in asiatischen Ländern, Schritt halten und sich verändern musste. So erfolgte eine Rückkehr in koloniale Muster: Der Süden nahm die schicksalshafte und unveränderbare Rolle des Rohstofflieferanten ein, der Norden jene des paternalistischen Almosengebers. Der freiwillige Aufpreis für Fair-Trade-Produkte nahm wiederum den christlichen Topos des gerechten Preises (iustum pretium) auf, der nicht im Wettbewerb zwischen Gleichberechtigten, sondern moralisch-

20 G. Kirsch: Neue Politische Ökonomie, 397. Historisch siehe R. Quaas: Fair Trade, 239, K. J. Kuhn: Entwicklungspolitische Solidarität, 419 oder M. Jennings: ,Almost an Oxfam in Itself‘. 
normativ gebildet wurde. ${ }^{21}$ Vergessen ging das von der ersten Ujamaa-Kampagne vorgebrachte Argument, der tansanische Pulverkaffee sei für den Schweizer Konsumenten billiger als Nescafé. ${ }^{22}$

Durch die Trennung der ursprünglichen Bewegung in professionelles Personal und passive Unterstützerinnen und Unterstützer der NGO veränderte sich auch die Vorstellung von der Rolle, welche die Schweizer Bevölkerung spielen sollte. Genau wie die professionellen NGOs in ihrem Diskurs Entwicklungsländer wieder zu passiven Idealkonstrukten zurückstuften, degradierten sie den politischen Bürger wieder zum passiven Spender und Konsumenten. Von der Schweizer Bevölkerung wurde nicht mehr erwartet, dass sie sich aktiv mit akademischer Handelstheorie auseinandersetzte und eigene Privilegien zugunsten der Bevölkerung in Entwicklungsländern aufgab. Die professionelle NGO setzte nun auf den kopflosen Bauchentscheid der Konsumentinnen und Konsumenten, die vor dem Supermarktregal aufgrund ihres schlechten Gewissens einen Fair-TradeAufpreis auswählen sollten. Die Nachfrage nach Fair Trade wurde von den professionellen NGOs, wie Anderson bemerkt, aktiv geschaffen. ${ }^{23}$ Erst das Vorhandensein der Fair-Trade-Produkte im Supermarktregal kreierte die Dichotomie zwischen fair und unfair, liess die Produkte ohne Gütesiegel als unfair erscheinen. Die ursprüngliche Idee der EvB-Arbeitsgruppe, ein „Schlechtezeichen“ einzuführen, hatte sich erübrigt. ${ }^{24}$

Betrachtet man die Landschaft der verschiedenen Schweizer Hilfswerke und Organisationen, war die Rolle der EvB als Think-Tank insbesondere in den 1970erJahren zentral. Denn von Seiten der akademischen Ökonomie war keinerlei Sukkurs für das neue Konzept zu erwarten, im Gegenteil: Indem die NGOs langsam eine paraakademische Lehre aufbauten, vertiefte sich der Graben zwischen der akademischen Ökonomie und der Fair-Trade- bzw. der Bio-Bewegung. Unterstützung erhielten Think-Tanks durch vereinzelte Wissenschaftlerinnen und Wissenschaftler anderer Fachbereiche - im Falle der EvB etwa durch den Politologen Gilbert Rist -, welche wiederum die Ökonomie als ideologische Pseudowissenschaft verurteilten und die Nichtbeachtung ökonomischer Studien wissenschaftlich guthiessen. ${ }^{25}$ Die konsequente Ausblendung der akademischen Ökonomie sowie der Aufbau eines paraakademischen Wissenschaftsbetriebs zum

21 H. D. Kurz: Geschichte des ökonomischen Denkens, 12-23.

22 SozArch, EvB, Ar 430.27.1. Kaffee Ujamaa 73-77: Plakat.

23 M. Anderson: Fair Trade: NGOs, 236.

24 SozArch, EvB, Ar 430.41.1. Textilien, Mappe 3: Koordinatorengruppe Aktion Globaler Supermarkt und Hunger. Aktion „Label“-Zeichen/Aktion Gütezeichen-Schlechtezeichen, Anne-Marie Holenstein, 30.5.1980, Fragenkatalog.

25 G. Rist: L'économie ordinaire. 
Thema Fair Trade war nötig, um in den 1970er- und 80er-Jahren das Paradox der zunehmenden Entwicklungshilfe und des abnehmenden Handels mit den ärmsten Ländern sowie das weitere Öffnen der Wohlstandsschere zu übertünchen. Allerdings machte auch die Wirtschaftskrise von 1973 die ursprüngliche Forderung der „Erklärung von Bern“, in der Schweiz Arbeitsplätze und Wirtschaftsleistung zugunsten von Entwicklungsländer aufzugeben, nun schwieriger. Die von den NGOs als Ersatz vorgebrachte Kritik an Tätigkeiten der multinationalen Konzerne mit Sitz in der Schweiz und Filialen in Entwicklungsländern hatte einen angenehmen Nebeneffekt: der implizite Einsatz für den Verbleib der Produktion und der Arbeitsplätze im Inland. Als der Glaube an die Möglichkeit eines alternativen Handelssystems allmählich und schleichend aufgegeben wurde, entstand zwischen NGOs und multinationalen Firmen, die nun zu Partnern wurden, überraschenderweise eine Symbiose. Die NGOs kritisierten zwar multinationale Konzerne, doch vermochten sie keine Alternative zu bieten, so dass sie anstatt des Aufbaus eines alternativen Handelssystems lediglich Auflagen an die Konzerne stellten. Damit lieferten sie den Grosskonzernen die westlichen Kunden- und Konsumentenwünsche frei Haus. $\mathrm{Zu}$ diesen Wünschen gehörte nicht nur die Zertifizierung von Endprodukten, sondern auch die Produktion im Inland. Malinowski und Sedlmaier nennen dies eine Verzahnung von Unternehmenskultur und Gegenkultur bis zur Ununterscheidbarkeit. ${ }^{26}$

Durch die Professionalisierung der NGOs hatte sich denn auch die Kritik verlagert. Nicht die eigene Nation, sondern supranationale Organisationen wurden jetzt kritisiert. Die Bewahrung der lokalen Produktion und der Arbeitsplätze im Inland wurde teilweise direkt, teilweise indirekt zu einem Hauptanliegen der NGOs; ein Anliegen, das sie sowohl in die nationale Politik wie zu den multinationalen Konzernen transportierten. Die Frage des Schaffens von Wachstum, von Arbeitsplätzen und Diversifikation in den Entwicklungsländern verschwand durch das Primat des Kleinbauern aus der Agenda. Der Arbeitslosigkeit in Entwicklungsländern, in den 1960er-Jahren das prädominante Thema in der Entwicklungsökonomie wie auch unter den frühen Aktivistinnen und Aktivisten, sollte, wenn sie in den späteren 1970ern überhaupt thematisiert wurde, mit dem Argument des Kleinbauerntums zu begegnen sein.

Nach einer Entstehungs- und Aufbauphase des NGO-Bereichs in den 1960erund 70er-Jahren fand nach etwa 1975 die professionelle Organisationsentwicklung und das Wachstum statt. Dies galt nicht nur für NGOs, sondern auch für den Alternativen Handel, der zunehmend kommerziell organisiert war. So schrieb etwa die Schweizer OS3 1979: „Um die Wirkung von OS3 zu verstärken, wird

26 S. Malinowski, A. Sedlmaier: ,1968‘ als Katalysator, 246. 
Wachstum, wird Ausweitung nötig sein. “27 Quaas beschreibt, wie auch die westdeutsche alternative Importgesellschaft GEPA zu Beginn der 1980er-Jahre sich Wachstum und die Erschliessung neuer Märkte zum Ziel setzte und sich in diesem Prozess auf einen von Kleinbauern in biologischem Landbau hergestellten Rohkaffee eingrenzte. ${ }^{28}$ Die faktische Entwicklung der NGOs und der von ihnen gegründeten Firmen stand somit in Widerspruch zur Wachstumskritik, die sie ab Mitte der 1970er-Jahre diskursiv äusserten. Mit dem Wachstum verbunden war, dass die nun professionell agierenden NGOs kommerzielle Dienstleistungen, etwa die Entwicklung von Lehrmitteln, anboten sowie Firmen wie etwa die OS3 gründeten. Hilton beobachtet: „many NGOs have developed from amateur and voluntarist roots to becoming highly professionalised and even business-oriented organisations. “29

Diese Entwicklung wurde gerade im Fall der EvB auch resigniert reflektiert. Das founder's syndrome setzte bei der EvB nach 1980 ein. Nachdem Strahm 1978 noch schwarze Zahlen hinterlassen hatte, fuhr insbesondere die Kampagne „Hunger ist ein Skandal“ Jahr für Jahr Verluste ein. ${ }^{30} 1982$ schlug ein Verlust von 86000 Franken zu Buche. ${ }^{31}$ Die idealistisch-utopische Arbeitsweise hatte zu Abnützungserscheinungen und Kündigungen geführt. 1983 waren die Aktivistinnen und Aktivisten der 1970er-Jahre nicht nur müde, sie standen auch vor dem finanziellen Kollaps und mussten rigorose Sparmassnahmen ergreifen, um die Organisation EvB zu retten. ${ }^{32} \mathrm{Al}$ Imfeld, der neben Rudolf Strahm zu den jungen Aktivisten der Anfangsjahre gehört hatte, fand klare Worte:

Eine Organisation wie die EvB wird trotz aller Ideale und heroischer Aufopferung der Mitarbeiter auf die Borstigkeit des Menschen-Tiers stossen. Vordergründig steht man Wortlaut zu den Prinzipien wie ,keine Arbeitsteilung,, ,Gleichstellung der inhaltlichen und administrativen Arbeit‘, ,Lohngleichheit‘, ,eigene Einschätzung der Bedürftigkeit‘, etc. Aber im Alltag treten Widersprüche auf. ${ }^{33}$

„Borstigkeit des Menschen-Tiers“ bedeutete in Al Imfelds poetischer Ausdrucksweise ein Eingeständnis dessen, was in der Politischen Ökonomie methodologi-

27 SozArch, Claro 1010.12. OS3 Gründung: Mario Carera, OS3 bald im breiteren Handel?, November 1979.

28 R. Quaas: Fair Trade, 240.

29 M. Hilton: A Historical Guide, 347.

30 SozArch, EvB, Ar 430.90.1. Jahresberichte 1978-2003. EvB: Jahresberichte 1979 bis 1984.

31 SozArch, EvB, Ar 430.90.1. Jahresberichte 1978-2003. EvB: Jahresbericht 1982, Revidierte Jahresrechnung der Erklärung von Bern, Deutsche Schweiz.

32 SozArch, EvB, Ar 430.90.1. Jahresberichte 1978-2003. EvB: Jahresbericht 1983, S. 14.

33 A. Imfeld: Die „Erklärung von Bern“ in der Krise. 
scher Individualismus genannt wird oder mit dem Sprichwort ,Jeder ist sich selbst am nächsten“ aus der römischen Literatur überliefert ist. ${ }^{34}$ Die einzelnen Angestellten hatten nach einer idealistischen Anfangsphase weder Kraft noch Lust, schlecht entlohnte Arbeit zu leisten. „Die EvB kennt zwei heikle Gebiete innerhalb des Sekretariats: Organisation der Arbeit und Entlohnung“, bestätigt Al Imfeld die Ergebnisse der britischen DANGO-Forschung auch für den Fall der EvB. ${ }^{35}$ Zudem war die EvB wie manche andere NGO zu dieser Zeit aufgrund der hohen Schulden schlicht gezwungen, die Organisation zu professionalisieren. ${ }^{36}$ Mit dem Begriff „Eigengesetzlichkeiten“ machte Al Imfeld wiederum eine Konzession an die Politische Ökonomie, welche ausgehend vom methodologischen Individualismus schlicht davon ausgeht, dass der Mensch sich als Individuum, als homo oeconomicus, verhalten wird und dass sich das Verhalten der Menschen im Kollektiv fast gesetzmässig voraussagen lässt. ${ }^{37}$ Dies versuchte Al Imfeld hier auszudrücken:

\begin{abstract}
Aber zwischen Kopf und Bauch geht da anderes vor. Wie soll sich jemand beim Lohn ehrlich und dennoch frei selbst einschätzen? Schämt sich der Idealist nicht seiner Forderung und schimpft tief innerlich im Geheimen doch über seine Ausbeutung? Ist es nicht falsch von gleichem Lohn zu reden in einem System, das anders gelagert ist? Erhält eine Bürokraft den landesüblichen Lohn und der Professionelle gleicht sich diesem Niveau an, dann haben eben nicht beide Seiten gleichviel beigetragen und die Last ist einseitig - und nicht gleich verteilt. Früher oder später entstehen Magengeschwüre und Kopfweh. ${ }^{38}$
\end{abstract}

„Magengeschwüre und Kopfweh“, dies war das founder's syndrome, die Standortbestimmung der Organisation EvB zu Beginn der 1980er-Jahre. Eine Konzession an die „Eigengesetzlichkeiten“ und die „Borstigkeit des Menschen-Tiers“ war unausweichlich, um die Organisation zu sanieren und zu retten.

\title{
5.2 Die Wachstumskritik überrollt Fair Trade: der Widerspruch wird übertüncht
}

Die Organisation EvB musste sich zu Beginn der 1980er-Jahre nochmals in einer weiteren Phase professionalisieren und war gezwungen, in Zukunft sowohl genug wie auch beständig Spenden einzunehmen. Die Konzentration ihres Konzepts der Nord-Süd-Gerechtigkeit auf eine Schnittmenge, in der sich eine möglichst breite

34 Siehe dazu erklärend G. Kirsch: Neue Politische Ökonomie, 19.

35 A. Imfeld: Die „Erklärung von Bern“ in der Krise.

36 N. J. Crowson, M. Hilton, J. McKay: NGOs in Contemporary Britain.

37 A. Imfeld: Die „Erklärung von Bern“ in der Krise.

38 Ebd. 
Schicht potentieller Spender traf, war somit zu Beginn der 1980er-Jahre die einzige Möglichkeit, die Organisation zu retten und zu erhalten. Doch wollte man den Wandel des Fair-Trade-Konzepts einzig durch die Professionalisierung der DritteWelt-Bewegung und als Reaktion auf die Präferenzen der Spenderinnen und Spender erklären, würde dies zu kurz greifen. Im Sinne Rodriks kann das seit den 1970er-Jahren beobachtete Paradox, Hilfe zu leisten statt Handel zu treiben, nicht allein durch die simple utilitaristische Vertretung von Eigeninteressen erklärt werden, sondern muss auch mit der Rolle der übergeordneten „Idee“ analysiert werden: ${ }^{39}$ Die ursprüngliche, von Prebisch und Singer vertretene dynamische Idee eines gerechten Nord-Süd-Handels wurde offensichtlich von der Wachstumskritik und vom Umweltschutz verdrängt. In der sozialen Bewegung im Umfeld der neu entstandenen NGOs wurde der Begriff „Nehmen“ allmählich uminterpretiert. In den UNCTAD-Jahren nach 1964 setzte sich innerhalb der Dritte-Welt-Bewegung für kurze Zeit eine Überlappung mit den Wirtschaftswissenschaften und dadurch eine positive Besetzung des Begriffs „Nehmen“ durch. Die Entwicklungsländer warfen dem Norden vor, nicht genug zu nehmen, nämlich einzig tropische Rohstoffe. Ziel, so die UNCTAD-Forderung, sollte ein „Mehr nehmen“ sein, daher auch die Forderung nach Trade, not Aid. In Zukunft sollten die westlichen Länder nicht nur tropische Rohstoffe, sondern sämtliche Rohstoffe sowie auch verarbeitete Produkte importieren. Allerdings hatte diese Forderung in der westlichen DritteWelt-Bewegung nicht Bestand. Schon etwa 1970, an der interkonfessionellen Konferenz im Bundeshaus, übte die schweizerische Dritte-Welt-Bewegung erstmals Kritik am „Nehmen“ von Fluchtgeldern aus Entwicklungsländern. Der EvBSlogan „Es kommt weniger darauf an, mehr zu geben, als weniger zu nehmen“ bezog sich zunächst einzig auf Fluchtgelder aus Entwicklungsländern und zielte auf den Finanzplatz Schweiz ab. ${ }^{40}$ Doch bereits im Rahmen der Welternährungskrise 1974 sollte der Aspekt des „Wegnehmens“ von Nahrung dazukommen. Ab Mitte der 1970er-Jahre verstand die Bewegung den Begriff „Nehmen“ allumfassend negativ. Ziel war die lokale, kleinbäuerliche, autarke Produktion, die mit dem Konsumverzicht einherging. Handel, der durch den Begriff „Nehmen“ gemeint war, erhielt eine negative Note. Damit war der Abschied von der akademischen Makroebene des internationalen Handels auch diskursiv besiegelt. Der Diskurs der NGOs betraf nun die Mikroebene des Konsums und der einzelnen Produkte. Die Kommerzialisierung und Annäherung an die Betriebswirtschaftslehre und an das Marketing ging einher mit dem Abschied von der Volkswirtschaftslehre und damit mit der Ablösung von der Forderung, dass die Industrie-

39 D. Rodrik: When Ideas Trump Interests.

40 A.-M. Holenstein-Hasler, R. Renschler, R. H. Strahm: Entwicklung heisst Befreiung, 37-38. 
staaten zugunsten der Entwicklungsländer auf protektionistische Privilegien verzichten sollten. ${ }^{41}$ Stattdessen begleitete nun eine paraakademische, statische Vorstellung von Nord-Süd-Gerechtigkeit die Kommerzialisierung des Fair Trade.

Das akademisch-volkswirtschaftliche UNCTAD-Konzept der 1960er-Jahre brachte, wie oben erwähnt, für professionelle NGOs Nachteile mit sich und liess sich schlecht verkaufen. Es beinhaltete den Imperativ, im Norden Privilegien aufzugeben, sah einen massiven Strukturwandel und somit grosse Veränderungen vor. Die Prebisch-Singer-These war kompliziert, akademisch, abstrakt und zu weit oben auf der Theorieebene. Dies machte das akademisch-ökonomische Konzept anstrengend und zeitraubend. Insbesondere aber bot es keine Möglichkeit, innert kurzer Zeit oder auch nur mittelfristig die eigenen westlichen Schuldgefühle abzubauen. Das neu entstandene wachstumskritische Konzept von Fair Trade, das zu Beginn der 1980er-Jahre „in Kraft trat“, brachte hingegen nur Vorteile. Es liess sich auf eine einfache, verständliche Botschaft reduzieren, die nun in ein Gütesiegel für Supermarktprodukte gefasst werden sollte. Es erforderte keine tiefgreifenden Veränderungen im Alltag und schon gar keinen umwälzenden Strukturwandel. Im Gegenteil, es trug eine konservative Botschaft in sich: Das Althergebrachte sollte bewahrt und Veränderungen sollten bekämpft werden. Insbesondere die Möglichkeit der Personifizierung eines Kleinbauern mit Foto oder Namen bot auch die Chance, Schuldgefühle in Form eines modernen Ablasshandels zu besänftigen. Das Konzept erwartete von der Bevölkerung nicht, dass sie denken oder sich engagieren sollte, sondern sah lediglich vor, dass die Konsumentinnen und Konsumenten zur richtigen Kaffeepackung greifen sollten. Mit dem Kauf bzw. dem ethisch korrekten Konsum war die Sühne abgeschlossen.

Der Fokus auf den Kleinbauern vermittelte der Spenderbasis, dass man sich für den Schutz indigener Kulturen einsetzte, brachte aber auch eine Rückkehr kolonialer Herablassung mit sich. Nach 1964 herrschte eine Aufbruchphase, in der die Dritte-Welt-Bewegung für den Süden und den Norden einen einheitlichen Wertmassstab anwandte. Interessanterweise wurde dieses Werte- und Weltbild von einer Gruppe Ökonomen vorgebracht, die oft entweder selbst aus dem Süden

41 Seit Beginn der 2000er-Jahre befasst sich der Bereich business ethics der Betriebswirtschaftslehre mit Fair Trade und benutzt diesen Begriff für den ethischen Konsum zertifizierter Produkte. Parallel dazu nutzen Volkswirtschafter den Begriff Fair Trade weiterhin zur Beschriebung gerechter Nord-Süd-Verhältnisse im Rahmen der WTO-Verhandlungen. Siehe dazu beispielhaft für business ethics etwa Andorfer, Veronika; Liebe, Ulf: Research on Fair Trade Consumption. A Review, in: Journal of Business Ethics 106/4 (2012) 415-435, zur Volkswirtschaft J. E. Stiglitz, A. Charlton: Fair Trade for All. 
oder aus Osteuropa stammten. ${ }^{42}$ Es galt implizit ein einheitliches Wertesystem der Moderne, mit dem Ziel einer einheitlichen Zivilisation bzw. Modernisierung durch Industrialisierung. Für die Dritte-Welt-Aktivisten im Westen war diese Sichtweise eine „Offenbarung“, wie Black schreibt: „It was a revelation. This was because 'living standards' had not been the window on these societies through which most observers had previously been looking. “43

Die Wirtschaftswissenschaften der 1960er-Jahre hatten den ursprünglich theologisch oder völkerkundlich geschulten Dritte-Welt-Aktivisten eine neue Sichtweise beschert. Für kurze Zeit war das Ziel der Dritte-Welt-Bewegung im Westen, dass die Länder und Menschen im Süden aufholen und zum Norden aufschliessen sollten. Dies bedingte, dass die Länder und Menschen im Norden ihre Privilegien aufgeben und beispielsweise Produkte aus Ländern des Südens auf ihren Märkten zulassen und kaufen sollten. Das Weltbild dieser kurzen Aufbruchphase der 1960er-Jahre kannte keinen Kulturrelativismus, sondern, wie gesagt, ein einheitliches Wertesystem. Interessanterweise brachte gerade diese Einheitlichkeit auch einen Aufruf zu massiven Strukturveränderungen im Norden und Süden mit sich. Eine „Revolution“ hatte Raúl Prebisch gefordert. ${ }^{44}$ Diese neue Sichtweise bedingte Offenheit und sehr viel Mut, aber auch den Willen zur Veränderung vor der eigenen Haustür. Es bedingte, dass Strukturen nicht mehr geschützt und bewahrt werden konnten, sondern völlig dem Einfluss der Entwicklung in anderen Weltregionen preisgegeben waren. Diese Vorstellung entsprach wahrlich einem Bild, das im Geiste der Aufbruchjahre vor 1968 entstanden war, einem Bild der Progressivität, der Dynamik, der grundsätzlichen, unvorhersehbaren Veränderung im Fluss. Es war die „Dynamik der Hoffnung“, wie Jacques Rossel schrieb, und Bob Dylan prophezeite: „The things they are a-changing“. ${ }^{45}$

Diese Vorstellung des Anspruchs aller Kulturen auf Wohlstand und Modernisierung muss diskutiert werden bezüglich ihrer Vor- und Nachteile für die Armutsbekämpfung. Der Vorteil dieser Vorstellung eines universellen Anspruchs auf Wohlstand war, dass nicht zwischen Nord und Süd unterschieden wurde: Kein als Wertepluralität getarnter Kulturrelativismus konnte eine Benachteiligung des Südens legitimieren. Ihr Nachteil war, dass die Modernisierung als absoluter Wert

42 Darunter Raúl Prebisch aus Argentinien, Celso Furtado aus Brasilien, Michael Kalecki aus Polen oder W. Arthur Lewis aus der karibischen Insel St. Lucia. Hans W. Singer war wiederum jüdisch und hatte aus Deutschland fliehen müssen. J. Toye, R. Toye: UN and Global Political Economy, 4-5.

43 M. Black: Oxfam the First 50 Years, 68.

44 Bodleian Library, Oxford. Oxfam archive, MS, Oxfam. COM/3/1/12. Folder 3: Haslemere Declaration, S. 4.

45 Siehe dazu Kapitel 1.2. Die Mission übt Selbstkritik: Jacques Rossel. 
gesetzt wurde. Der kulturelle Wert lange gewachsener volkswirtschaftlicher Strukturen und die Bedeutung dieser Strukturen für den Einzelnen und die Gesellschaft hatten keinen Platz in der Gleichung. Sowohl im Süden wie im Norden sollte die Modernisierung althergebrachte Strukturen grundlegend und ungehemmt zerstören. Diese Radikalität war der Aufbruchsphase der 1960er-Jahre zu eigen, und sie wurde sowohl von den linken Vertreterinnen und Vertretern der Prebisch-Singer-These wie von der radikal-neoliberalen Chicagoer School geprägt. Auch wurde sie von der aufgeschlossenen, christlich-bürgerlichen Mitte in der Schweiz, wie hier gezeigt wurde, vorsichtig portiert. Die althergebrachten, kolonialen Strukturen in Ländern des Südens wurden sowohl von Vertretern aus dem Süden, wie Prebisch, als auch von der westlichen Dritte-Welt-Bewegung als ungerecht und daher als nicht schützenswert empfunden. Die Strukturen im Norden galten ebenfalls als kolonial und deshalb nicht als bewahrenswert. Doch diese Schumpetersche Haltung der schöpferischen, ja heilsam-erlösenden Zerstörung sollte von kurzer Dauer sein. ${ }^{46}$ Die schweizerische Dritte-Welt-Bewegung begann nur einige Jahre nach dem Manifest von 1968, den kulturellen Wert der schweizerischen Landwirtschaft $\mathrm{zu}$ entdecken und sich für diesen einzusetzen. Gleichzeitig wurde das Modernisierungsparadigma auf internationaler Ebene durch den Slogan Small is beautiful in Frage gestellt. Die Antithese zum Modernisierungsparadigma war somit, dass sowohl im Norden wie im Süden gewisse volkswirtschaftliche Strukturen, allen voran die Landwirtschaft, ihren eigenen, kulturellen Wert hatten und nicht der Zerstörung im Zuge der Modernisierung anheimfallen sollten.

Die Wertepluralität, die „pluralité des mondes“, wurde zum Argument für die Erhaltung herkömmlicher Strukturen und das Aufhalten der Modernisierung im Süden. ${ }^{47}$ Der Zivilisationspfad der Industrieländer wurde relativiert und nicht als einzig gültiger Wertmassstab genommen. Die „pluralité des mondes“ galt als Argument gegen den Ethnozentrismus. Die Frage stellt sich jedoch, inwiefern diese Wertepluralität tatsächlich eine Antithese oder Synthese war - bzw. inwiefern sie nicht einfach ein Rückschritt zu bestehenden kolonialen Denkmustern bedeutete. Denn die Aufwertung herkömmlicher volkswirtschaftlicher Strukturen in Ländern des Südens aus kulturellen Gründen hob den Imperativ der Modernisierung auf. Mit dem Argument der kulturellen Pluralität konnte jegliche volkswirtschaftliche Differenz zwischen Norden und Süden legitimiert werden: Aus Armut wurde Kultur.

46 H. D. Kurz: Geschichte des ökonomischen Denkens, 84.

47 P. Bungener, J. Grinevald: La pluralité des mondes. 
Die Abkehr von der abstrakten, unpersönlichen Volkswirtschaft und die Hinwendung zur Kultur transportierte zudem das Bild des Kleinbauern in die westliche Dritte-Welt-Bewegung. Dieses Stereotyp war, wie gezeigt, auch marketingstrategisch von Nutzen. Kuhn beobachtet etwa für die „Hunger ist ein Skandal“-Aktion der EvB, im Rahmen welcher Anne-Marie Holenstein erstmals die Idee eines Gütesiegels lancierte, eine marketingorientierte Spendensammlung, die sich an karitativen Motiven orientierte. ${ }^{48}$ Bleibt man bei der Argumentation der Marketingstrategie, lässt sich beobachten, dass der Kleinbauer keine Neueinführung war, sondern eine Rückkehr zum altbekannten Marketing der Missionen symbolisierte. Die Bilder von Kleinbauern auf den Broschüren und Packungen der Fair-Trade-Produkte gleichen auffällig verschiedenen Figuren auf sogenannten „Nickneger“-Kassen, die bis in die 1960er-Jahre bei den christlichen Missionen in Gebrauch waren. Auch die personalisierten Geschichten von Kleinbauern in den Broschüren der Hilfswerke, die Fair Trade unterstützten, können durchaus als Weiterführung der Missionsliteratur gesehen werden. Tatsächlich ersetzte die Zeitschrift „Der Auftrag“ fliessend die langjährige Zeitschrift der Basler Mission, den „Evangelischen Heidenboten“. ${ }^{49}$ Es müsste genauer untersucht werden, wie und ob sich die Fair-Trade-Bilder von Kleinbauern von der bibliothèque coloniale unterscheiden, der nicht die Andersartigkeit, sondern die Überlegenheit der westlichen Zivilisation und teilweise der weissen Rasse zugrunde lag.

Auch die Rückkehr zum Primat der Umwelt - die Vorrang hat vor dem Menschen - kann als Rückkehr in koloniale Muster interpretiert werden. Dabei standen nicht etwa die negativen Folgen der Umweltverschmutzung für die Bevölkerung der Dritten Welt auf dem Plan. Ziel des Umweltschutzes der Dritte-WeltBewegung der 1970er-Jahre war die Umwelt an sich und vor Ort: die Luft, der Wald, die Pflanzen und insbesondere die Tiere. Auf eigentümliche Weise begann die Fair-Trade-Bewegung in den 1970er-Jahren, den Tierschutz in ihr Programm aufzunehmen..$^{50}$ Fast ruckartig wurde der Einsatz für die politisch souveränen Staaten im Süden, die auf dem Weg zur wirtschaftlichen Gleichstellung mit dem Norden waren, mit jenem gegen Schweinemasten und Legehennenfabriken in Westeuropa vertauscht. Die Menschen in der Dritten Welt und ihr an der UNCTAD geäussertes Bedürfnis nach Wirtschaftswachstum schienen bedeutungslos geworden zu sein. Einzig der Kleinbauer hatte als Bewahrer der Biodiversität noch

48 K. J. Kuhn: Entwicklungspolitische Solidarität, 398.

49 Kooperation evangelischer Kirchen und Missionen, Verein Mission gemeinsam tun, Mission 21: Auftrag.

50 So finanzierte etwa der Tierschutzverband eine von der EvB vertriebene Studie, A.-M. Holenstein, J. Davis: Zerstörung durch Überfluss, 5. 
seine berechtigte Funktion. Die Stadt, die der EvB-Erstunterzeichner Jacques Rossel 1967 noch als Ort der Schönheit und der Hoffnung besungen hatte, wurde unter dem Einfluss der Wachstumskritik zum Moloch, vor dem die Bevölkerung der Dritten Welt bewahrt werden sollte.

\section{3 „Déjeuner en paix“: der Rückzug ins Private oder das Ende eines Protestzeitalters}

Die Autoren des DANGO-Projekts, die den Aufstieg der NGOs in Grossbritannien nach 1945 untersucht haben, kommen zu einem interessanten Schluss. Sie stellen den Aufstieg der privaten NGOs in den Kontext der Privatisierung unter der neoliberalen Premierministerin Margaret Thatcher. Sie stellen fest, dass NGOs in dieser Zeit zu technokratischen Expertenorganisationen wurden, die auf privater Basis soziale Dienstleistungen anboten, die der Wohlfahrtsstaat zwar wünschte, aber aufgrund der neoliberalen Politik nicht selbst bereitstellte. ${ }^{51}$ Diesen Aufstieg der technokratischen und sehr professionellen Expertenorganisationen stellten die Autoren der Studie wiederum in den Kontext der Politikverdrossenheit, was mit dem Ende des Protestzeitalters und dem Rückzug ins Private zusammenfiel. Sie stellten fest, dass der Protest bzw. die politische Einmischung von den NGOs übernommen wurde. Diese kreierten „The Protest Business“ und nahmen der Bevölkerung gegen Bezahlung eines Mitgliederbeitrags oder gar einer einmaligen Spende die Mühen des nächtelangen Diskutierens, der Proteste und der politischen Einmischung ab. ${ }^{52}$

Eine ähnliche Entwicklung fand in der Schweiz statt. ${ }^{53}$ Der Rückzug ins Private zeichnete sich bereits zu Beginn der 1970er-Jahre ab. Neu gegründete, landwirtschaftliche Kommunen auf dem Land boten den abgekämpften Aktivisten eine Rückzugsmöglichkeit von den Protesten auf den Strassen der Grossstadt. ${ }^{54}$ In einem weiteren Schritt ergab sich durch den Konsum der entsprechenden ökologischen und fairen Produkte eine noch einfachere und bequemere Möglichkeit, ökologische und solidarische Anliegen umzusetzen. Die NGOs übernahmen somit den aktiven Part des Protestes, lenkten diesen aber in Bahnen. Die NGO selbst schwächte ihre Positionen ab, schwenkte auf eine Mitteposition ein oder entzog sich geradezu der politischen Diskussion. Kuhn beobachtet für

51 M. Hilton: Politics of Expertise.

52 Ebd., 55, A. G. Jordan, W. A. Maloney: The Protest Business.

53 B. Mahler: ,umdenken - umschwenken', 79.

54 S. Bittner: Jenseits der Kleinfamilie, 26. 
das Jahrzehnt der 1980er, dass entwicklungspolitische Organisationen wie die EvB bezüglich der politischen Dimensionen ihrer Tätigkeit zurückhaltender wurden. ${ }^{55}$ Während die Angestellten der NGO EvB in den 1970er-Jahren durchaus am Gesetzgebungsprozess teilgenommen und diesen verfolgt hatten, wandten sie sich nun nicht mehr direkt an die Schweizer Bürgerinnen und Bürger als politische Subjekte, sondern an die Konsumentinnen und Konsumenten. Die Pulverkaffee-Aktion stellte hier einen ersten Übergang dar, es folgten die Jutesack-Aktion (welche gar nicht mehr auf die Handelspolitik hinwies), Aktionen zum Tourismus (der ökonomisch gesehen ebenfalls ein Konsumgut darstellt) sowie Ende der 1970er-Jahre Kochbücher. Unmerklich übernahm die Dritte-Welt-Bewegung den Fokus auf den Konsum, den sie eigentlich kritisierte. ${ }^{56}$ Stimmig liess sich gemäss Malinowski und Sedlmaier die Kulturrevolution der 68er in den Wandlungsprozess des postindustriellen Kapitalismus einordnen. ${ }^{57}$

Die ökologischen und entwicklungspolitischen Überlegungen in der konstanten Beschäftigung mit dem eigenen Konsumverhalten verschleierten dabei die Widersprüchlichkeit. Die Beschäftigung mit dem fairen Handel war vom Kopf in den Bauch gerutscht. Die politische Bewusstseinsbildung wich der Beeinflussung der unbewussten Konsumentscheide. Auch bezüglich jener Bereiche, in denen die EvB zu Beginn der 1980er-Jahre weiterhin die politische Bürgerin bzw. den Bürger ansprach, stellte sie fest, dass ihren Anstrengungen kaum Erfolg beschieden würde, wenn sie die Bürger nicht über die „Gefühlsebene“ - und somit über das Bauchgefühl - ansprechen konnte. ${ }^{58}$ In einer Standortbestimmung zur zukünftigen Arbeit der EvB im Bereich Wirtschaftspolitik, der ja ursprünglich das ausschliessliche Thema des Manifests gewesen war, wird mit Ernüchterung konstatiert:

Allerdings scheinen die Aktions- bzw. die Mobilisierungsmöglichkeiten auf dem Gebiete der Wirtschaftspolitik ungemein viel schwieriger. Der Anschluss an die unmittelbare Gefühls-

55 K. J. Kuhn: Entwicklungspolitische Solidarität, 398.

56 B. C. Schär, R. Ammann, T. Färber, S. Bittner, M. Hofer, Y. Niederhäuser, V. Sperisen, M. Griesshammer, R. Fischer, M. Schär, R. Schär, E. Vaudan: Bern 68, 8.

57 S. Malinowski, A. Sedlmaier: ,1968‘ als Katalysator, 239.

58 In einer empirischen Studie von 2007 haben Psychologen belegt, dass Identifikation mit Opfern und eine entsprechende Spendebereitschaft über die Gefühlsebene in stärkerem Masse erfolgt als über Information in Form von statistischen Daten. Siehe dazu Small, Deborah A.; Loewenstein, George; Slovic, Paul: Sympathy and callousness. The impact of deliberative thought on donations to identifiable and statistical victims, in: Organizational Behavior and Human Decision Processes 102/2 (2007) 143-153. 
ebene oder die eigene Praxis (wie bei Ernährung, Lektüre, Tourismus, usw.) ist nicht so direkt machbar. $^{59}$

Auch örtlich hatte eine Verlagerung stattgefunden: Die Schweiz sollte nicht mehr an der Grenze, in der Zollpolitik, „Privilegien aufgeben“, einzig im Dritte-WeltLaden und bald schon im Supermarkt sollten die Konsumentinnen und Konsumenten einen kleinen Aufpreis bezahlen. Auch die ausführliche Informationsarbeit, die „Bewusstseinsbildung“, die ursprünglich das wichtigste Ziel des Verkaufs von Fair-Trade-Produkten wie dem Ujamaa-Kaffee und der Jutetasche ausgemacht hatte, wurde auf ein kleines wortloses Symbol im Supermarkt reduziert. Damit wandelte sich auch die Rolle des Dritte-Welt-Ladens. Dieser wurde vom wichtigen Versammlungsort und Resonanzraum der Bewegung zum überflüssigen und ineffizienten Tante-Emma-Laden zurückgestuft. Die Aufgaben der Weltläden als Informationsvermittler übernahmen in den 1980er-Jahren die professionellen NGOs. Noch zu Beginn der 1970er-Jahre hatte der Raum des Weltladens an sich eine bewusstseinsbildende Funktion erfüllt:

Ideally, the shop would offer not just the possibility to obtain goods and information, but also provide a space for debate, meetings, and a duplicating machine. A typical monthly bulletin published by a world shop in the 1970s included information on the shop's activities and informed readers about issues concerning the global economy and the situation of developing countries. ${ }^{60}$

Der Weltladen war nur solange nötig, wie die Bewegung noch bewusst eine ehrenamtlich-aktivistische Graswurzelbewegung war. Mit der Verlagerung des Aktivismus hin zu bezahlten Angestellten von NGOs fiel die Notwendigkeit des Weltladens als dezentraler Begegnungsraum weg.

Die 1980er-Jahre sahen eine Aufteilung der Dritte-Welt-Bewegung in kommerzielle, in Staat und Gesellschaft eingebettete NGOs auf der einen Seite und kleine, gewaltbereite Gruppierungen auf der anderen Seite. Auch in der Schweiz waren zu Beginn der 1980er-Jahre Sachbeschädigungen und Hausbesetzungen an der Tagesordnung. Ursprung mancher Sachbeschädigungen und Anschläge der 1980er-Jahre war auch der Protest gegen ungerechte wirtschaftliche Nord-SüdBeziehungen. ${ }^{61}$

59 SozArch, EvB, Ar 430.11.5. Entwurf für die Weiterarbeit im Bereich der Wirtschaftspolitik, Termin: Mai -bis Ende 1984.

60 Dam, Peter Van: Moralizing Postcolonial Consumer Society, 235.

61 K. Karcher: Sisters in Arms, K. Karcher: Violence. 
Das starke Schuldgefühl der 68er-Generation, das die Auseinandersetzung mit der Nord-Süd-Frage und die Bestrebungen hin zu einem gerechten Handel mit dem Süden angetrieben hatte, war in den 1980er-Jahren einer Alternativlosigkeit und Anspruchshaltung gewichen. Sehr schön spiegelt sich die Mentalität der Politikverdrossenheit und des Rückzugs ins Private im Song „Déjeuner en paix“ wider, der 1991 herauskam und die 1980er-Jahre atmosphärisch einfing. ${ }^{62}$ Der von Stephan Eicher gesungene und vom französischen Schriftsteller Philippe Djian stammende Text handelt von einem Mann, der beim Frühstück die Zeitung liest und darauf wartet, dass seine Geliebte aufwacht. Als sie zum Frühstück kommt, möchte sie nichts über den Zustand der Welt wissen, sondern ,in Frieden frühstücken“. Die Politikverdrossenheit der 1980er-Jahre hatte die Bahn frei gemacht für die Kommerzialisierung der Fair-Trade-Produkte. Der ab 1992 in Schweizer Supermärkten erhältliche, fair zertifizierte Kaffee und bald darauf auch Zucker, Honig, Schokoladenpulver oder Orangensaft erlaubten es der Generation der 1980er-Jahre, fortan ihr Frühstück in Frieden zu geniessen. ${ }^{63}$

Vor allem aber setzte zu Beginn der 1980er-Jahre eine „Müdigkeit“ ein, die der Politikverdrossenheit Vorschub leistete. Al Imfeld fing die Stimmung sprachlich exakt ein:

Werden die Engagierten ihres Bewusstseins müde? Kann jemand wie die EvB die Bürgerklagen auf nimmermüde Weise durchalten? Und kann man sich nicht mit Kampf gegen den Hunger übersättigen? Zeitgeist: Müdigkeit. ${ }^{64}$

Auch die NZZ zitierte 1981 genüsslich Vertreter der Dritte-Welt-Bewegung, die Leuten in den eigenen Reihen vorwarfen, sie seien „müde, ängstlich und lendenlahm geworden“. ${ }^{65}$ Die Resignation und Müdigkeit der Schweizer Dritte-WeltBewegung zu Beginn der 1980er-Jahre ordnete sich wiederum ein in einen europaweit spürbaren Verlust der elektrisierenden Betroffenheit von 1968 und in den Übergang in eine gleichgültige Pseudopolitisiertheit. Der junge französische Philosoph Pascal Bruckner fing diese Stimmung 1983 mit seinem Essay „Le sanglot de l'homme blanc“ ein. ${ }^{66}$ Ausgerechnet bei Jean Zieglers Hausverlag Seuil in Paris, der 1976 Zieglers „Une Suisse au-dessus de tout soupçon“ gedruckt hatte, erschien Bruckners Dekonstruktion der Dritte-Welt-Bewegung mit ihrem hausge-

62 Siehe dazu Stephan Eichers Album „Engelberg“ (1991).

63 Siehe dazu etwa auf der Webseite des Schweizer Grossverteilers Coop die Rubrik „Labels“ (http://www.coop.ch/de/labels/guetesiegel/max-havelaar/philosophie.html) (13.9.2017).

64 A. Imfeld: Die „Erklärung von Bern“ in der Krise.

65 C. W., „Soziale Befreiung“ gegen Entwicklungszusammenarbeit, NZZ, 29.5.1981.

66 P. Bruckner: Le sanglot. 
machten, zutiefst europäisch-christlich diffusen Schuldgefühl. Das Jahr 1983 stellte sich sozusagen als Negativ des Jahres 1968 heraus, indem Bruckner, als wende er sich direkt an die EvB, für eine nochmalige Neufassung des Solidaritätsbegriffs plädierte. Er warf den Dritte-Welt-Aktivistinnen und -Aktivisten der vergangenen Jahre vor, sie seien weinende Kinder mit unreflektierten Schuldgefühlen gegenüber dem Süden. Und er forderte die Dritte-Welt-Bewegung auf, erwachsen zu werden und - wie es auch Jacques Rossel mit Rückgriff auf Max Weber später formulieren sollte - Verantwortung zu übernehmen, denn: „Le tiersmondisme, on l'a vu, entretient une confusion constante entre culpabilité et responsabilité [...].“67

Auch die Dritte-Welt-Bewegung der Bundesrepublik Deutschland verlor gemäss Ruben Quaas Mitte der 1980er-Jahre Visionen und Utopien. Wie Quaas beobachtet, hatte auch in Deutschland die Dependenztheorie an Rückhalt verloren, das Umweltproblem machte Wirtschaftswachstum nicht mehr tragfähig und es mangelte an Entwürfen, wie das globale Wohlstandsgefälle zu überwinden sei. ${ }^{68}$ Während die Dritte-Welt-Bewegung müde, abgenutzt, losgesagt von den akademischen Wirtschaftswissenschaften und gefangen in Bruckners Schuldgefühlen eine Metamorphose in professionelle NGOs mit klar formulierten Aufgaben in der Dienstleistungsgesellschaft der 1980er-Jahre durchmachte, nahm gemäss Toye und Toye gleichzeitig der Nord-Süd-Dialog, wie er in den 1960er-Jahren fordernd und hoffnungsvoll begonnen hatte, auch auf der Bühne der internationalen Handelsvereinbarungen ein Ende. ${ }^{69}$ Entwicklungsländer wie Tansania, die in den 1960er- und zu Beginn der 1970er-Jahre für die Dritte-Welt-Aktivistinnen und -Aktivisten der Industrieländer Sehnsuchtsorte gewesen waren und einen dritten Weg dargestellt hatten - und im Fall von Tansania zahlreiche junge Menschen mit „Tanzaphilia“ angesteckt hatten -, waren zu Beginn der 1980er-Jahre verarmt, hochverschuldet und politisch instabil. ${ }^{70}$ Gleichsam wandelte sich die Sahara, bis in die 1970er-Jahre „Sehnsuchtsort für westliche Zivilisationskritiker“, zum Synonym für Dürre und Hungerkatastrophen. ${ }^{71}$ Die Aufbruchstimmung, die kurz nach der Unabhängigkeit den selbstbewussten und fordernden Nord-Süd-Dialog getragen hatte, machte einer Verdrossenheit aufgrund der gescheiterten Wirtschaftspolitik Platz. ${ }^{72}$ In den Informationstexten der OS3 wird die Hoffnung in

67 Ebd., 293.

68 R. Quaas: Fair Trade, 271.

69 J. Toye, R. Toye: UN and Global Political Economy, 4.

70 Ebd., 257.

71 F. Rauh: Tierkadaver im Wüstensand, 155.

72 R. Quaas: Fair Trade, 270, macht dieselbe Beobachtung für die Bundesrepublik Deutschland. 
Tansania aufgegeben und das Experiment eines afrikanischen Sozialismus als gescheitert betrachtet, auch wenn die Firma den tansanischen Pulverkaffee weiterhin im Sortiment führte. ${ }^{73}$ Auch die ärmsten Entwicklungsländer, wovon die meisten auf dem afrikanischen Kontinenten $\mathrm{zu}$ finden waren, verlegten sich darauf, Hilfe zu verlangen. Die Möglichkeit, innerhalb des GATT- oder des UNSystems Marktzugang einzufordern, nahmen sie nicht mehr wahr und wurden zum ,sleeping dog“. ${ }^{74}$

73 K. J. Kuhn: Fairer Handel und Kalter Krieg, 41. Heute wird derselbe Pulverkaffee unter dem neuen Namen Baraza geführt, ebd., 40.

74 M. Elsig, P. Stucki: Low-Income Developing Countries. 